American Journal of Biochemistry and Biotechnology 4 (2): 208-217, 2008

ISSN 1553-3468

(C) 2008 Science Publications

\title{
Evidence of Mitochondrial Dysfunction in Autism and Implications for Treatment
}

\author{
Daniel A. Rossignol, J. Jeffrey Bradstreet \\ International Child Development Resource Center, 3800 W. Eau Gallie Blvd., Suite 105, \\ Melbourne, FL 32934
}

\begin{abstract}
Classical mitochondrial diseases occur in a subset of individuals with autism and are usually caused by genetic anomalies or mitochondrial respiratory pathway deficits. However, in many cases of autism, there is evidence of mitochondrial dysfunction (MtD) without the classic features associated with mitochondrial disease. $\mathrm{MtD}$ appears to be more common in autism and presents with less severe signs and symptoms. It is not associated with discernable mitochondrial pathology in muscle biopsy specimens despite objective evidence of lowered mitochondrial functioning. Exposure to environmental toxins is the likely etiology for MtD in autism. This dysfunction then contributes to a number of diagnostic symptoms and comorbidities observed in autism including: cognitive impairment, language deficits, abnormal energy metabolism, chronic gastrointestinal problems, abnormalities in fatty acid oxidation, and increased oxidative stress. MtD and oxidative stress may also explain the high male to female ratio found in autism due to increased male vulnerability to these dysfunctions. Biomarkers for mitochondrial dysfunction have been identified, but seem widely under-utilized despite available therapeutic interventions. Nutritional supplementation to decrease oxidative stress along with factors to improve reduced glutathione, as well as hyperbaric oxygen therapy (HBOT) represent supported and rationale approaches. The underlying pathophysiology and autistic symptoms of affected individuals would be expected to either improve or cease worsening once effective treatment for MtD is implemented.
\end{abstract}

Keywords: mitochondria, inflammation, oxidative stress, antioxidants, hyperbaric oxygen therapy, lactate, ammonia, Co-Enzyme Q10

\section{INTRODUCTION}

Autism and its related spectrum of disorders (ASD) are neurodevelopmental disorders characterized by substantial impairments in social interaction, difficulty with communication, and restrictive and repetitive behaviors ${ }^{[1]}$. However, this psychologically defined cluster of symptoms and behaviors represents a broad phenotypic expression which may result from numerous underlying biological mechanisms and genetic vulnerabilities interacting with environmental factors ${ }^{[2]}$. Three rigorously performed epidemiological studies demonstrate that the prevalence of autism has increased in recent years ${ }^{[3-5]}$. It is currently estimated that autism affects as many as 1 out of 150 children (67 per 10,000) in the United States ${ }^{[3,6]}$. However since autism occurs four times as frequently in males as females ${ }^{[7]}$, reporting the prevalence in children will underestimate the numbers of affected males significantly. A reasonable extraction of the overall data when applied to the male population finds 1 in 93 males (54 per
10,000) are likely affected with autism. This represents an extraordinarily high prevalence of autism and should generate concern about environmental factors that are more likely to adversely affect males.

Mitochondrial disorders, once thought to be rare, are now regarded as one of the most common metabolic diseases in children ${ }^{[8]}$. Classical mitochondrial diseases occur in a subset of autism cases and are usually caused by genetic anomalies or mitochondrial respiratory pathway abnormalities ${ }^{[9-15]}$. These diseases often result in severe symptoms including: limb weakness, cardiac conduction defects and cardiomyopathy, ataxia, and stroke-like episodes ${ }^{[8]}$. They have also been associated with developmental regression and growth retardation in $\mathrm{ASD}^{[16-18]}$ including one boy who lost language skills, had hyperactivity, abnormal social interaction, restricted interests, stereotypical behaviors, seizures, and self-injurious behaviors ${ }^{[16]}$. However, within a significant subset of ASD, studies reveal evidence of mitochondrial dysfunction (MtD) without the classic features associated with mitochondrial disease ${ }^{[9,16,19,20]}$.

Corresponding Author: $\quad$ Daniel A. Rossignol, International Child Development Resource Center, 3800 W. Eau Gallie Blvd., Suite 105, Melbourne, FL 32934, Tel: 321-259-7111 
For example, one study of 25 children with autistic-type features including developmental delay, seizures, and hypotonia found no mitochondrial structural changes upon muscle biopsy. These children did not fit any known categories of mitochondrial encephalomyopathies $^{[20]}$.

MtD in autism was first suggested by overlapping symptoms of both conditions and led Lombard to hypothesize that autism may be caused by $\mathrm{MtD}^{[21]}$. In comparison to classical mitochondrial disease, $\mathrm{MtD}$ occurs more commonly in autism ${ }^{[22]}$, is not as severe in symptomatology, and is not associated with any discernable mitochondrial abnormality upon muscle biopsy. It is, however, associated with laboratory evidence of lowered mitochondrial functioning. This $\mathrm{MtD}$ is likely precipitated by environmental toxins, and could contribute to a number of diagnostic symptoms and comorbidities of autism including: cognitive impairment, language deficits, abnormal energy metabolism, chronic gastrointestinal problems, abnormalities in fatty acid oxidation, and increased oxidative stress. Although biomarkers for mitochondrial deficiency have been identified, it appears that MtD is significantly under-recognized in autism, and a widespread under-prescribing of potentially beneficial therapies often occurs. Interestingly, MtD has been implicated in other chronic neurological diseases, including Alzheimer's disease ${ }^{[23,}{ }^{24]}$, Parkinson's disease ${ }^{[25]}$, amyotrophic lateral sclerosis ${ }^{[25]}$, Huntington's disease ${ }^{[26]}$, and Friedreich's ataxia ${ }^{[26]}$. This may be due to the fact that the aerobic metabolic demand of the brain is very high, making it extraordinarily vulnerable to any decline in mitochondrial efficiency ${ }^{[19]}$, largely due to the extraordinary dependence of synaptic transmission on high energy output from mitochondria ${ }^{[27]}$.

\section{MtD in Autism}

Evidence of MtD in autism: One of the first studies suggesting $\mathrm{MtD}$ in autism used ${ }^{31} \mathrm{P}$ nuclear magnetic resonance spectroscopy (MRS) to investigate energy metabolism in the brain of individuals with autism by measuring steady-state levels of phosphocreatine (PCr), adenosine triphosphate (ATP), adenosine diphosphate (ADP), and inorganic orthophosphate and comparing these levels to neurotypical individuals. The study found that $\mathrm{PCr}$ was lower in the autism group, consistent with "increased utilization of PCr to maintain brain ATP levels," and this finding was correlated with language and neuropsychological problems. The group with autism also had lower levels of esterified endproducts $(\alpha \mathrm{ATP}, \quad \alpha \mathrm{ADP}, \quad$ dinucleotides, and diphosphosugars). The authors speculated that the clinical abnormalities found in autism may be secondary events that result from these "alterations in brain bioenergetics"[28]. A subsequent study also confirmed evidence of "altered energy metabolism" in the brains of children with autism ${ }^{[29]}$. These studies are consistent with impaired mitochondrial function and document the fact that some individuals with autism have overall lowered cellular energetics and deficient reserve mitochondrial energy capacity that could lead to cognitive impairment, language deficits, and abnormal energy metabolism.

Impairments in mitochondrial function can also deplete glutathione levels ${ }^{[30]}$ and lead to chronic gastrointestinal (GI) problems ${ }^{[19]}$ because the GI tract is highly dependent on glutathione to function properly ${ }^{[31]}$. Several studies demonstrate that chronic GI problems are commonly found in autism ${ }^{[32,33]}$ including chronic constipation $^{[34]}$. Constipation is frequently observed in patients with mitochondrial disorders ${ }^{[35]}$ and the chronic constipation found in some ASD cases may be a manifestation of MtD. Seizures ${ }^{[36]}$ and hypotonia ${ }^{[37]}$ in autistic individuals may also be manifestations of MtD.

Abnormalities in fatty acid oxidation may also reflect underlying $\mathrm{MtD}$ in autistic individuals. Fatty acid oxidation is noted in autism ${ }^{[38]}$, as well as impairments in beta oxidation and oxidative phosphorylation associated with carnitine deficiency and reduced production of phospholipids containing 22:6n-3 fatty acids (docosahexaenoic acid) ${ }^{[39]}$. For example, in one case, a child with autism and hypotonia, but with normal muscle strength, demonstrated elevated blood lactate, low bicarbonate, and an abnormal acyl-carnitine profile. This child was eventually diagnosed with a dysfunction of mitochondrial beta oxidation ${ }^{[40]}$. In another study of 15 autistic children, compared to 18 control mentally retarded children, the autism population displayed a "marked reduction" in 22:6n-3 levels ${ }^{[41]}$. Taken together, these findings suggest that MtD may be common in autism and that the specific changes described in this section may be diagnostic of this effect.

MtD and oxidative stress in autism: During mitochondrial respiration, an inability to neutralize reactive oxygen species and free radicals leads to oxidative stress ${ }^{[23,42]}$. Oxidative damage in humans is thought to contribute to aging and neurological diseases $^{[43]}$. The inner membrane of the mitochondria contains a large amount of free radical scavengers including glutathione, vitamin $\mathrm{C}$, and vitamin $\mathrm{E}$, as well as anti-oxidant enzymes such as superoxide dismutase 
$(\mathrm{SOD})^{[26]}$. Animal studies demonstrate that mice that are deficient in SOD have molecular defects in mitochondrial respiration, and that improvement of symptoms occur with administration of $\mathrm{SOD}^{[44]}$. Some children with autism have lower levels of reduced glutathione ${ }^{[45,46]}$ and $\mathrm{SOD}^{[47]}$; both could lead to mitochondrial damage and dysfunction ${ }^{[48]}$. Impairments in mitochondrial function lead to further oxidative stress $^{[49]}$ and continued lower glutathione levels ${ }^{[30]}$, and a vicious negative cycle can ensue. Since increased oxidative stress is commonly found in autism $^{[50]}$, this harmful cyclical process is likely to persist. In a pivotal autism study, plasma oxidized glutathione, a reliable marker of intracellular oxidative stress ${ }^{[51]}$, was $72 \%$ higher in children with autism when compared to neurotypical children ${ }^{[45]}$. Furthermore, decreased activities of certain antioxidant enzymes including $\mathrm{SOD}^{[47]}$, glutathione peroxidase ${ }^{[47]}$, catalase $^{[52]}$, and paraoxonase ${ }^{[53]}$ are found in some individuals with autism. Some ASD children also express increased lipid peroxidation ${ }^{[52,54]}$, including increased malondialdehyde, which is a marker of oxidative stress and lipid peroxidation ${ }^{[55]}$. One group of researchers has actually suggested that "oxidative stress (endogenous or environmental) may contribute to the development and clinical manifestations of autism",[46]. As an example, in one study, clinical regression (loss of previously acquired skills) in some autistic children was associated with lower levels of serum antioxidant enzymes $^{[55]}$.

Oxidative stress may also play a role in the increased male to female ratio observed in autism. Because of lower levels of reduced glutathione, mitochondria from males, when compared to females, are more vulnerable to oxidative stress ${ }^{[56]}$. In animal studies, due to lower enzyme activity levels of SOD and glutathione peroxidase in males, oxidative damage to mitochondrial DNA is 4-fold higher in males compared to females ${ }^{[56]}$. Oxidative stress, as measured by a loss in tissue ascorbate after cerebral ischemia, is greater in the brain of male animals compared to females ${ }^{[57]}$. The activity of catalase, an anti-oxidant enzyme, is also higher in females than in males ${ }^{[58]}$, and estrogen induces glutathione peroxidase activity ${ }^{[59]}$. In one animal model, in cerebral arteries, estrogen attaches to estrogen receptor- $\alpha$ and increases mitochondrial capacity for ATP production by increasing mitochondrial enzyme activity including citrate synthase and complex IV. Estrogen also decreases production of hydrogen peroxide thereby reducing oxidative stress ${ }^{[60]}$. Therefore, males with increased oxidative stress may manifest more MtD and more severe clinical symptoms when compared to females.
Biomarkers of classical mitochondrial disease and MtD in autism: Classical mitochondrial diseases are commonly associated with abnormal laboratory findings including: elevated lactate, ammonia, aspartate aminotransferase, pyruvate, and creatine kinase, and lowered carnitine levels. These markers can be useful in evaluating mitochondrial diseases ${ }^{[20]}$. However, many of these laboratory abnormalities have also been described in autistic individuals with $\mathrm{MtD}$ and without any other evidence of classical mitochondrial disease. For example, elevated blood lactate levels consistent with MtD have been described in numerous autistic individuals $^{[29,40,61-65]}$, sometimes affecting as many as $43 \%$ of children with autism studied when compared to neurotypical children ${ }^{[17]}$. In one study, ASD children with an elevated blood lactate level underwent deltoid muscle biopsy and approximately $35 \%$ (5 out of 14) were found to have classical mitochondrial disease ${ }^{[22]}$. However, this study is significant because although some of the children with autism and elevated lactate had a true mitochondrial disease, most had normal muscle biopsies which were felt to be consistent with MtD.

In several studies of individuals presenting with autism, but without classical mitochondrial disease, increased ammonia has also been described; this led some authors to conclude that MtD was present ${ }^{[40,63,64]}$. Typically, these ammonia levels are not nearly as elevated as those that have been observed in some conditions that can cause autism, such as urea cycle disorders ${ }^{[66]}$. Ammonia requires three ATP molecules to be converted into urea and in animal studies, elevated ammonia can lead to brain ATP depletion ${ }^{[67]}$. Therefore, the increased ammonia noted in autism may be both consequentially and causally related to ATP depletion. One study of 100 children with autism and a history of clinical regression, but without classical mitochondrial disease, found evidence of MtD that was expressed by significantly reduced levels of free and total carnitine and increased ammonia, alanine, and lactate blood levels ${ }^{[64]}$.

Other markers of MtD that are elevated in autism without evidence of classical mitochondrial disease include pyruvate $\mathrm{e}^{[17]}$ and creatine kinase ${ }^{[18]}$. One study of 159 children with autism, compared to 94 neurotypical children, demonstrated elevations in aspartate aminotransferase and creatine kinase suggestive of possible defects in oxidative phosphorylation. The authors concluded that "further metabolic evaluation is indicated in autistic patients and that defects of oxidative phosphorylation might be prevalent." They also commented that MtD occurring during early childhood would lead to additional 
oxidative stress that could impair the body's ability to handle certain infections or immune activation from immunizations received during that time ${ }^{[18]}$.

Possible causes of MtD in autism: The cause of MtD in some autistic individuals is unknown but could be a secondary manifestation resulting from exposure to certain environmental toxins. These agents could directly damage the mitochondria and reduce their efficiency, or impair functional activity by increasing body burden of free radicals and resultant oxidative stress. Drugs like valproic acid and thalidomide ${ }^{[68,69]}$, as well as exposure to viruses such as rubella and cytomegalovirus $^{[70,71]}$ are examples of environmental factors that have a known causal role in autism. However, many more environmental toxins including pesticides $^{[53,72]}$, polychlorinated biphenyls (PCB's) ${ }^{[73]}$, industrial chemicals ${ }^{[73-76]}$, and toxic metals such as $\operatorname{mercury}^{[77,78]}$, lead $^{[79,80]}$, cadmium, nickel ${ }^{[74]}$, and tin ${ }^{[81]}$ have also been implicated in ASD. Notably, many of these toxins including mercury ${ }^{[82,83]}$, arsenic, cadmium, lead $^{[84]}$, pesticides ${ }^{[85]}$, and industrial chemicals ${ }^{[86]}$ inhibit mitochondrial function. In fact, mitochondria may be the earliest target of metal neurotoxicity ${ }^{[82]}$. Other substances may also hinder mitochondrial function. Propionic acid is produced by Clostridia in the gastrointestinal tract and impairs mitochondrial function by directly inhibiting oxidative phosphorylation $^{[87]}$. Higher amounts of clostridia are typically present in the gastrointestinal tract of autistic individuals, and injection of propionic acid into rats has recently been shown to produce an autistic-like illness ${ }^{[87]}$. By increasing oxidative stress ${ }^{[49]}$ and lowering glutathione levels ${ }^{[30]}$, MtD may actually lead to problems in detoxifying environmental contaminants $^{[46]}$ and consequently increase susceptibility to metal toxicity ${ }^{[88]}$.

Testing autistic individuals for MtD: Based upon these findings, some researchers have proposed that mitochondrial disorders may be the most common metabolic cause of autism ${ }^{[10,65]}$ and that MtD should be suspected and tested for in children with autism who have associated neurological findings or a family history of mitochondrial problems ${ }^{[14,22]}$. Certain biomarkers may be useful for evaluating possible MtD in autistic individuals. Some researchers suggest pyruvate levels should be measured during routine evaluation of ASD children ${ }^{[61]}$. However, measuring a pyruvate level is not always practical because typically it is only available in teaching or specialty hospitals. The plasma alanine to lysine ratio (normal range is 1.5-2.5) is a surrogate marker for pyruvate; a ratio above 2.5 is consistent with an elevation in pyruvate ${ }^{[18]}$. A lactate level is "a simple, non-invasive, low-cost means for the initial diagnostic approach" of mitochondrial problems ${ }^{[8]}$. Mitochondrial problems can also be screened for by examining urinary organic acids ${ }^{[19]}$. Assessing carnitine and ammonia levels ${ }^{[64]}$ and oxidative stress markers may also be useful. Screening for any of these blood and urine tests may be helpful in identifying MtD in ASD.

\section{Potential Treatments of MtD in Autism}

Nutritional supplements and antioxidants: Treatment of oxidative stress with antioxidants and other nutritional supplements may ameliorate $\mathrm{MtD}$ in some individuals with autism ${ }^{[26]}$. In animal studies, acetyl-Lcarnitine restored partial mitochondrial function and muscle activity ${ }^{[89]}$. Coenzyme Q10 administration in rats increased mitochondrial concentrations, extended survival times, and exhibited neuroprotective effects ${ }^{[90]}$. In human studies, coenzyme Q10 improved symptoms in patients with mitochondrial disease. For instance, in one patient with mitochondrial disease and coenzyme Q10 blood concentrations below $25 \%$ of normal, coenzyme Q10 administration led to subjective improvements ${ }^{[91]}$. In another study of 2 patients with mitochondrial encephalomyopathy, administration of coenzyme Q10 and idebenone led to clinical improvements as measured by EEG and standardized psychological testing. Lactate and pyruvate levels also decreased after this treatment ${ }^{[92]}$. In one double-blind, crossover study of 8 patients with mitochondrial encephalomyopathy, coenzyme Q10 improved fatigue and muscle exercise endurance, and decreased serum lactate and pyruvate levels ${ }^{[93]}$. Thiamine, pyridoxine, and riboflavin may also improve mitochondrial function $^{[21]}$. In one study, pyridoxine improved language, affection, and behavior in 3 out of 8 autistic children with $\mathrm{MtD}^{[62]}$. Ginkgo biloba extract also appears to lessen oxidative stress in mitochondria ${ }^{[94]}$. Furthermore, treatment with antioxidants to decrease oxidative stress may improve mitochondrial function and energy production ${ }^{[95]}$. In fact, treatment with antioxidants in ASD children raised the levels of reduced glutathione in the serum and appeared to improve symptoms ${ }^{[45]}$. In one double-blind study, the use of vitamin $\mathrm{C}$ improved behavior in autistic individuals when compared to a placebo ${ }^{[96]}$. Once MtD is identified, treatments with these nutritional supplements may lead to improvements in mitochondrial function and reductions of negative symptoms. 
Hyperbaric oxygen therapy (HBOT): Several studies have described cerebral hypoperfusion in autism ${ }^{[97-99]}$ and this decreased blood flow has been reported to correlate with core autistic symptoms ${ }^{[100]}$ and could lead to relative cerebral hypoxia. In fact, several studies have demonstrated evidence of hypoxia in the brains of some individuals with autism as measured by a reduction in brain $\mathrm{Bcl}-2$ and an increase in brain $\mathrm{p} 53^{[101}$, 102]. Elevated p53 is induced by hypoxia ${ }^{[103]}$ and a decrease in Bcl-2 is associated with increased apoptosis provoked by hypoxia ${ }^{[104]}$. Hypoxia can impair mitochondrial function ${ }^{[105]}$ and diminish ATP production ${ }^{[106]}$. Since only approximately $0.3 \%$ of inhaled oxygen is ultimately delivered to mitochondria ${ }^{[107]}$, increasing the oxygen delivery to mitochondria by hyperbaric oxygen therapy (HBOT) may actually improve mitochondrial function ${ }^{[108,109]}$. For example, in a mouse model with an intrinsic impairment of mitochondrial complex IV, HBOT at 2 atmospheres (atm) "significantly ameliorate[d] mitochondrial dysfunction" and delayed the onset of motor neuron disease when compared to control mice ${ }^{[108]}$. In animal studies, HBOT has been shown to increase the amount of work done by mitochondria ${ }^{[110]}$, improve mitochondrial function after brain injury ${ }^{[109]}$, and prevent mitochondrial deterioration ${ }^{[111]}$ when compared to room air pressure and oxygen levels. HBOT also increased sperm motility by augmenting mitochondrial oxidative phosphorylation in fructolysis-inhibited sperm cells. Fructose is the sugar used by sperm for energy production $^{[112]}$. Furthermore, the use of HBOT prevented apoptosis and improved neurological recovery after cerebral ischemia by opening mitochondrial ATP-sensitive potassium channels ${ }^{[113]}$. In one animal model, hypoxia and ischemia led to diminished ATP and phosphocreatine production; the addition of HBOT restored these levels to near-normal and increased energy utilization when compared to room air oxygen and pressure levels ${ }^{[106]}$. Finally, HBOT has recently been shown to activate mitochondrial DNA transcription and replication, and increase the biogenesis of mitochondria in the brains of animals $^{[114]}$. One small case series suggested that HBOT may improve symptoms in some autistic children ${ }^{[115]}$.

\section{CONCLUSION}

Classical mitochondrial diseases affect a small number of individuals with autism and typically occur due to genetic anomalies and mitochondrial respiratory chain deficits. However, MtD, a condition that does not have the classic features of mitochondrial disease, is found among some individuals with autism and is associated with abnormal biomarkers of mitochondrial function. A variety of environmental toxins could be disrupting both the structure and function of the mitochondria in these individuals, and these toxins should be considered in the evaluation of children with autism. MtD may contribute to a number of symptoms and comorbidities observed in autism including: cognitive impairment, language deficits, abnormal energy metabolism, chronic gastrointestinal problems, abnormalities in fatty acid oxidation, and increased oxidative stress. Additionally, since there is increased vulnerability of males to these dysfunctions, $\mathrm{MtD}$ may provide an explanation as to why the high male to female ratio in autism exists. MtD can be diagnosed via certain biomarkers and is potentially treatable. Therefore, increased efforts at MtD screening of all individuals affected with autism should be encouraged. Potentially, there are a large number of children with autism affected by $\mathrm{MtD}$, and since the possibility of available treatments for this aspect of autism exist, investigation of methodologies for correcting the underlying mechanisms of MtD should be urgently undertaken.

\section{ACKNOWLEDGEMENTS}

The authors would like to thank Mr. Michael Haynes and Dr. Lauren Underwood for insightful comments and help with editing of the manuscript.

\section{REFERENCES}

1. American Psychiatric Association, 1994. Diagnostic and Statistical Manual of Mental Disorders, 4th ed. Washington, DC: American Psychiatric Press.

2. Herbert, M.R, 2005. Autism: a brain disorder, or a disorder that affects the brain? Clin. Neuropsych., 2:354-379.

3. Bertrand, J., A. Mars, C. Boyle, F. Bove, M. Yeargin-Allsopp, P. Decoufle, 2001. Prevalence of autism in a United States population: the Brick Township, New Jersey, investigation. Pediatrics, 108:1155-1161.

4. Chakrabarti, S., E. Fombonne, 2001. Pervasive developmental disorders in preschool children. JAMA, 285:3093-3099.

5. Baird, G., T. Charman, S. Baron-Cohen, A. Cox, J. Swettenham, S. Wheelwright, A. Drew, 2000. A screening instrument for autism at 18 months of 
age: a 6-year follow-up study. J. Am. Acad. Child Adolesc. Psychiatry, 39:694-702.

6. Centers for Disease Control and Prevention, 2007. Prevalence of Autism Spectrum Disorders Autism and Developmental Disabilities Monitoring Network, Six Sites, United States, 2000. M.M.W.R., 56:1-40.

7. Bailey, A., W. Phillips, M. Rutter, 1996. Autism: towards an integration of clinical, genetic, neuropsychological, and neurobiological perspectives. J. Child. Psychol. Psychiatry, 37:89126.

8. Zeviani, M., B. Bertagnolio, G. Uziel, 1996. Neurological presentations of mitochondrial diseases. J. Inherit. Metab. Dis., 19:504-520.

9. Filiano, J.J., M.J. Goldenthal, C.H. Rhodes, J. Marin-Garcia, 2002. Mitochondrial dysfunction in patients with hypotonia, epilepsy, autism, and developmental delay: HEADD syndrome. J. Child Neurol., 17:435-439.

10. Lerman-Sagie, T., E. Leshinsky-Silver, N. Watemberg, D. Lev, 2004. Should autistic children be evaluated for mitochondrial disorders? J. Child Neurol., 19:379-381.

11. Ramoz, N., J.G. Reichert, C.J. Smith, J.M. Silverman, I.N. Bespalova, K.L. Davis, J.D. Buxbaum, 2004. Linkage and association of the mitochondrial aspartate/glutamate carrier SLC25A12 gene with autism. Am. J. Psychiatry, 161:662-669.

12. Segurado, R., J. Conroy, E. Meally, M. Fitzgerald, M. Gill, L. Gallagher, 2005. Confirmation of association between autism and the mitochondrial aspartate/glutamate carrier SLC25A12 gene on chromosome 2q31. Am. J. Psychiatry, 162:21822184.

13. Sue, C.M., C. Bruno, A.L. Andreu, A. Cargan, J.R. Mendell, C.Y. Tsao, M. Luquette, J. Paolicchi, S. Shanske, S. DiMauro, D.C. De Vivo, 1999. Infantile encephalopathy associated with the MELAS A3243G mutation. J. Pediatr., 134:696700.

14. Pons, R., A.L. Andreu, N. Checcarelli, M.R. Vila, K. Engelstad, C.M. Sue, D. Shungu, R. Haggerty, D.C. De Vivo, S. DiMauro, 2004. Mitochondrial DNA abnormalities and autistic spectrum disorders. J. Pediatr., 144:81-85.

15. Serajee, F.J., H. Zhang, A.H.M.M Huq, 2006. Prevalence of common mitochondrial point mutations in autism. Neuropediatrics, 37(Suppl 1):S127.

16. Graf, W.D., J. Marin-Garcia, H.G. Gao, S. Pizzo, R.K. Naviaux, D. Markusic, B.A. Barshop, E.
Courchesne, R.H. Haas, 2000. Autism associated with the mitochondrial DNA G8363A transfer RNA(Lys) mutation. J. Child Neurol., 15:357361.

17. Laszlo, A., E. Horvath, E. Eck, M. Fekete, 1994. Serum serotonin, lactate and pyruvate levels in infantile autistic children. Clin. Chim. Acta., 229:205-207.

18. Poling, J.S., R.E. Frye, J. Shoffner, A.W. Zimmerman, 2006. Developmental regression and mitochondrial dysfunction in a child with autism. J. Child Neurol., 21:170-172.

19. Nissenkorn, A., A. Zeharia, D. Lev, A. FatalValevski, V. Barash, A. Gutman, S. Harel, T. Lerman-Sagie, 1999. Multiple presentations of mitochondrial disorders. Arch. Dis. Child, 81:209214.

20. Marin-Garcia, J., R. Ananthakrishnan, M.J. Goldenthal, J.J. Filiano, H.B Sarnat, 1999. Skeletal muscle mitochondrial defects in nonspecific neurologic disorders. Pediatr. Neurol., 21:538-542.

21. Lombard, J., 1998 Autism: a mitochondrial disorder? Med. Hypotheses, 50:497-500.

22. Oliveira, G., L. Diogo, M. Grazina, P. Garcia, A. Ataide, C. Marques, T. Miguel, L. Borges, A.M. Vicente, C.R. Oliveira, 2005. Mitochondrial dysfunction in autism spectrum disorders: a population-based study. Dev. Med. Child Neurol., 47:185-189.

23. Ohta, S., I. Ohsawa, 2006. Dysfunction of mitochondria and oxidative stress in the pathogenesis of Alzheimer's disease: on defects in the cytochrome c oxidase complex and aldehyde detoxification. J. Alzheimers Dis., 9:155-166.

24. Manczak, M., T.S. Anekonda, E. Henson, B.S. Park, J. Quinn, P.H. Reddy, 2006. Mitochondria are a direct site of $\mathrm{A}$ beta accumulation in Alzheimer's disease neurons: implications for free radical generation and oxidative damage in disease progression. Hum. Mol. Genet., 15:1437-1449.

25. Martin, L.J., 2006. Mitochondriopathy in Parkinson disease and amyotrophic lateral sclerosis. J Neuropathol. Exp. Neurol., 65:11031110 .

26. Trushina, E., C.T. McMurray, 2007. Oxidative stress and mitochondrial dysfunction in neurodegenerative diseases. Neuroscience, 145:1233-1248.

27. Billups, B., I.D. Forsythe, 2002. Presynaptic mitochondrial calcium sequestration influences transmission at mammalian central synapses. J. Neurosci., 22:5840-5847. 
28. Minshew, N.J., G. Goldstein, S.M. Dombrowski, K. Panchalingam, J.W. Pettegrew, 1993. A preliminary 31P MRS study of autism: evidence for undersynthesis and increased degradation of brain membranes. Biol. Psychiatry, 33:762-773.

29. Chugani, D.C., B.S. Sundram, M. Behen, M.L. Lee, G.J. Moore, 1999. Evidence of altered energy metabolism in autistic children. Prog. Neuropsychopharmacol. Biol. Psychiatry, 23:635641.

30. Sherer, T.B., R. Betarbet, A.K. Stout, S. Lund, M. Baptista, A.V. Panov, M.R. Cookson, J.T. Greenamyre, 2002. An in vitro model of Parkinson's disease: linking mitochondrial impairment to altered alpha-synuclein metabolism and oxidative damage. J. Neurosci., 22:7006-7015.

31. Hoensch, H., I. Morgenstern, G. Petereit, M. Siepmann, W.H. Peters, H.M Roelofs, W. Kirch, 2002. Influence of clinical factors, diet, and drugs on the human upper gastrointestinal glutathione system. Gut, 50:235-240.

32. Uhlmann, V., C.M. Martin, O. Sheils, L. Pilkington, I. Silva, A. Killalea, S.B. Murch, J. Walker-Smith, M. Thomson, A.J. Wakefield, J.J. O'Leary, 2002. Potential viral pathogenic mechanism for new variant inflammatory bowel disease. Mol. Pathol., 55:84-90.

33. Furlano, R.I., A. Anthony, R. Day, A. Brown, L. McGarvey, M.A. Thomson, S.E. Davies, M. Berelowitz, A. Forbes, A.J. Wakefield, J.A. Walker-Smith, S.H Murch, 2001. Colonic CD8 and gamma delta T-cell infiltration with epithelial damage in children with autism. J. Pediatr., 138:366-372.

34. Valicenti-McDermott, M., K. McVicar, I. Rapin, B.K. Wershil, H. Cohen, S. Shinnar, 2006. Frequency of gastrointestinal symptoms in children with autistic spectrum disorders and association with family history of autoimmune disease. J. Dev. Behav. Pediatr., 27(2 Suppl):S128S136.

35. Chinnery, P.F., D.M. Turnbull, 1997. Mitochondrial medicine. Q.J.M., 90:657-667.

36. Peake, D., L.M. Notghi, S. Philip, 2006. Management of epilepsy in children with autism. Current Paediatrics, 16:489-494.

37. Rapin, I., 1997. Autism. N. Engl. J. Med., 337:97-104.

38. Bell, J.G., E.E. MacKinlay, J.R. Dick, D.J. MacDonald, R.M. Boyle, A.C. Glen, 2004. Essential fatty acids and phospholipase A2 in autistic spectrum disorders. Prostaglandins Leukot. Essent. Fatty Acids, 71:201-204.
39. Infante, J.P., V.A. Huszagh, 2000. Secondary carnitine deficiency and impaired docosahexaenoic (22:6n-3) acid synthesis: a common denominator in the pathophysiology of diseases of oxidative phosphorylation and betaoxidation. FEBS Lett., 468:1-5.

40. Clark-Taylor, T., B.E. Clark-Taylor, 2004. Is autism a disorder of fatty acid metabolism? Possible dysfunction of mitochondrial betaoxidation by long chain acyl-CoA dehydrogenase. Med. Hypotheses, 62:970-975.

41. Vancassel, S., G. Durand, C. Barthelemy, B. Lejeune, J. Martineau, D. Guilloteau, C. Andres, S. Chalon, 2001. Plasma fatty acid levels in autistic children. Prostaglandins Leukot. Essent. Fatty Acids, 65:1-7.

42. Reddy, P.H., 2006. Amyloid precursor proteinmediated free radicals and oxidative damage: implications for the development and progress of Alzheimer's disease. J. Neurochem., 96:1-13.

43. Singh, K.K., 2006. Mitochondrial damage checkpoint, aging, and cancer. Ann. N.Y. Acad. Sci. 1067:182-190.

44. Nojiri, H., T. Shimizu, M. Funakoshi, O. Yamaguchi, H. Zhou, S. Kawakami, Y. Ohta, M. Sami, T. Tachibana, H. Ishikawa, H. Kurosawa, R.C. Kahn, K. Otsu, T. Shirasawa, 2006. Oxidative stress causes heart failure with impaired mitochondrial respiration. J. Biol. Chem., 281:33789-33801.

45. James, S.J., P. Cutler, S. Melnyk, S. Jernigan, L. Janak, D.W. Gaylor, J.A. Neubrander, 2004. Metabolic biomarkers of increased oxidative stress and impaired methylation capacity in children with autism. Am. J. Clin. Nutr., 80:1611-1617.

46. James, S.J., S. Melnyk, S. Jernigan, M.A. Cleves, C.H. Halsted, D.H. Wong, P. Cutler, K. Bock, M. Boris, J.J. Bradstreet, S.M. Baker, D.W. Gaylor, 2006. Metabolic endophenotype and related genotypes are associated with oxidative stress in children with autism. Am. J. Med. Genet. B. Neuropsychiatr. Genet., 141:947-956.

47. Yorbik, O., A. Sayal, C. Akay, D.I. Akbiyik, T. Sohmen, 2002. Investigation of antioxidant enzymes in children with autistic disorder. Prostaglandins Leukot. Essent. Fatty Acids, 67:341-343.

48. Cadenas, E., K.J. Davies, 2000. Mitochondrial free radical generation, oxidative stress, and aging. Free Radic. Biol. Med., 29:222-230.

49. Albers, D.S., M.F. Beal, 2000. Mitochondrial dysfunction and oxidative stress in aging and 
neurodegenerative disease. J. Neural. Transm. Suppl., 59:133-154.

50. Chauhan, A., V. Chauhan, 2006. Oxidative stress in autism. Pathophysiology, 13:171-181.

51. Dickinson, D.A., H.J. Forman, 2002. Glutathione in defense and signaling: lessons from a small thiol. Ann. N. Y. Acad. Sci., 973:488-504.

52. Zoroglu, S.S., F. Armutcu, S. Ozen, A. Gurel, E. Sivasli, O. Yetkin, I. Meram, 2004. Increased oxidative stress and altered activities of erythrocyte free radical scavenging enzymes in autism. Eur. Arch. Psychiatry Clin. Neurosci., 254:143-147

53. D’Amelio, M., I. Rica, R. Sacco, X. Liu, L. D'Agruma, L.A. Muscarella, V. Guarnieri, R. Militerni,. C. Bravaccio, M. Elia, C. Schneider, R. Melmed, S. Trillo, T. Pascucci, S. Puglisi-Allegra, K.L. Reichelt, F. Macciardi, J.J. Holden, A.M Persico, 2005. Paraoxonase gene variants are associated with autism in North America, but not in Italy: possible regional specificity in geneenvironment interactions. Mol. Psychiatry, 10:1006-1016.

54. Yao, Y., W.J. Walsh, W.R. McGinnis, D. Pratico, 2006. Altered vascular phenotype in autism: correlation with oxidative stress. Arch. Neurol., 63:1161-1164.

55. Chauhan, A., V. Chauhan, W.T. Brown, I. Cohen, 2004. Oxidative stress in autism: increased lipid peroxidation and reduced serum levels of ceruloplasmin and transferrin - the antioxidant proteins. Life Sci., 75:2539-2549.

56. Borras, C., J. Sastre, D. Garcia-Sala, A. Lloret, F.V. Pallardo, J. Vina, 2003. Mitochondria from females exhibit higher antioxidant gene expression and lower oxidative damage than males. Free Radic. Biol. Med., 34:546-552.

57. Kume-Kick, J., D.C. Ferris, I. Russo-Menna, M.E. Rice, 1996. Enhanced oxidative stress in female rat brain after gonadectomy. Brain Res., 738:8-14.

58. Carrillo, M.C., S. Kanai, Y. Sato, K. Kitani, 1992. Age-related changes in antioxidant enzyme activities are region and organ, as well as sex, selective in the rat. Mech. Ageing Dev., 65:187198.

59. Ha, E.Jj, A.M. Smith, 2003. Plasma selenium and plasma and erythrocyte glutathione peroxidase activity increase with estrogen during the menstrual cycle. J. Am. Coll. Nutr., 22:43-51.

60. Stirone, C., S.P. Duckles, D.N. Krause, V. Procaccio, 2005. Estrogen increases mitochondrial efficiency and reduces oxidative stress in cerebral blood vessels. Mol. Pharmacol., 68:959-965.

61. Coleman, M., J.P. Blass, 1985. Autism and lactic acidosis. J. Autism Dev. Disord., 15:1-8.

62. Moreno, H., L. Borjas, A. Arrieta, L. Saez, A. Prassad, J. Estevez, E. Bonilla, 1992. [Clinical heterogeneity of the autistic syndrome: a study of 60 families]. Invest. Clin., 33:13-31.

63. Filipek, P.A., J. Juranek, M. Smith, L.Z. Mays, E.R. Ramos, M. Bocian, D. Masser-Frye, T.M. Laulhere, C. Modahl, M.A. Spence, J.J Gargus, 2003. Mitochondrial dysfunction in autistic patients with $15 q$ inverted duplication. Ann. Neurol., 53:801-804.

64. Filipek, P.A., J. Juranke, M.T. Nguyen, C. Cummings, J.J. Gargus, 2004. Relative carnitine deficiency in autism. J. Autism Dev. Disord., 34:615-23.

65. Correia, C., A.M. Coutinho, L. Diogo, M. Grazina, C. Marques, T. Miguel, A. Ataide, J. Almeida, L. Borges, C. Oliveira, G. Oliveira, A.M. Vicente, 2006. Brief report: High frequency of biochemical markers for mitochondrial dysfunction in autism: no association with the mitochondrial aspartate/glutamate carrier SLC25A12 gene. J. Autism Dev. Disord.,36:1137-1140.

66. Görker, I., U. Tüzün, 2005. Autistic-like findings associated with a urea cycle disorder in a 4-yearold girl. J. Psychiatry Neurosci., 30:133-135.

67. Kosenko, E., Y. Kaminsky, E. Grau, M.D. Minana, G. Marcaida, S. Grisolia, V. Felipo, 1994. Brain ATP depletion induced by acute ammonia intoxication in rats is mediated by activation of the NMDA receptor and $\mathrm{Na}+, \mathrm{K}(+)$-ATPase. J. Neurochem., 63:2172-2178.

68. Rodier, P.M., S.L. Hyman, 1998. Early environmental factors in autism. Mental Retard. Dev. Disabil. Res. Rev., 4:121-128.

69. Stromland, K., V. Nordin, M. Miller, B. Akerstrom, C. Gillberg, 1994. Autism in thalidomide embryopathy: a population study. Dev. Med. Child Neurol., 36:351-356.

70. Chess, S., P. Fernandez S. Korn, 1978. Behavioral consequences of congenital rubella. J. Pediatr., 93:699-703.

71. Yamashita, Y., C. Fujimoto, E. Nakajima, T. Isagai, T. Matsuishi, 2003. Possible association between congenital cytomegalovirus infection and autistic disorder. J. Autism Dev. Disord., 33:455459.

72. Worth, J., 2002. Paraoxonase polymorphisms and organophosphates. Lancet, 360:802-803. 
73. Edelson, S.B., D.S. Cantor, 1998. Autism: xenobiotic influences. Toxicol. Ind. Health, 14:799-811.

74. Windham, G.C., L. Zhang, R. Gunier, L.A. Croen, J.K. Grether, 2006. Autism spectrum disorders in relation to distribution of hazardous air pollutants in the San Francisco bay area. Environ. Health Perspect., 114:1438-1444.

75. Edelson, S.B., D. Cantor, 2000. The neurotoxic etiology of the autistic spectrum disorders: a replication study. Toxicol. Indust. Health, 16:239247.

76. Grandjean, P., P.J. Landrigan, 2006. Developmental neurotoxicity of industrial chemicals. Lancet, 368:2167-2178.

77. Bernard, S., A. Enayati, L. Redwood, H. Roger, T. Binstock, 2001. Autism: a novel from of mercury poisoning. Med. Hypotheses, 56:462-471.

78. Palmer, R.F., S. Blanchard, Z. Stein, D. Mandell, C. Miller, 2006. Environmental mercury release, special education rates, and autism disorder: an ecological study of Texas. Health Place, 12:203209.

79. Eppright, T.D., J.A. Sanfacon, E.A. Horwitz, 1996. Attention deficit hyperactivity disorder, infantile autism, and elevated blood-lead: a possible relationship. Missouri Medicine, 93:136-138.

80. Lidsky, T.I., J.S. Schneider, 2005. Autism and autistic symptoms associated with childhood lead poisoning. J. Appl. Research, 5:80-87.

81. Swartzwelder, H.S., W. Holahan, R.D. Myers, 1983. Antagonism by d-amphetamine of trimethyltin-induced hyperactivity evidence toward an animal model of hyperkinetic behavior. Neuropharmacology, 22:1049-1054.

82. Yee, S., B.H. Choi, 1996. Oxidative Stress in neurotoxic effects of methylmercury poisoning. NeuroToxicology, 17:17-26.

83. Shenker, B.J., T.L. Guo, I. O, I.M. Shapiro, 1999. Induction of apoptosis in human T-cells by methyl mercury: temporal relationship between mitochondrial dysfunction and loss of reductive reserve. Toxicol. Appl. Pharmacol., 157:23-35.

84. Pulido, M.D., A.R. Parrish, 2003. Metal-induced apoptosis: mechanisms. Mut. Res., 533:227-241.

85. Yamano, T., S. Morita, 1995. Effects of pesticides on isolated rat hepatocytes, mitochondria, and microsomes II. Arch. Environ. Contam. Toxicol., 28:1-7.

86. Hiura, T.S., N. Li, R. Kaplan, M. Horwitz, J.C. Seagrave, A.E. Nel, 2000. The role of a mitochondrial pathway in the induction of apoptosis by chemicals extracted from diesel exhaust particles. J. Immunol., 165:2703-2711.

87. MacFabe, D.F., D.P. Cain, K. Rodriguez-Capote, A.E. Franklin, J.E. Hoffman, F. Boon, A.R. Taylor, M. Kavaliers, K.P. Ossenkopp, 2007. Neurobiological effects of intraventricular propionic acid in rats: possible role of short chain fatty acids on the pathogenesis and characteristics of autism spectrum disorders. Behav. Brain Res., 176:149-169.

88. Kern, J.K., A.M. Jones, 2006. Evidence of toxicity, oxidative stress, and neuronal insult in autism. J. Toxicol. Environ. Health. B Crit. Rev., 9:485-499.

89. Hagen, T.M., R.T. Ingersoll, C.M. Wehr, J. Lykkesfeldt, V. Vinarsky, J.C. Bartholomew, M.H. Song, B.N Ames, 1998. Acetyl-L-carnitine fed to old rats partially restores mitochondrial function and ambulatory activity. Proc. Natl. Acad. Sci. U.S.A., 95:9562-9566.

90. Matthews, R.T., L. Yang, S. Browne, M. Baik, M.F. Beal, 1998. Coenzyme Q10 administration increases brain mitochondrial concentrations and exerts neuroprotective effects. Proc. Natl. Acad. Sci. U.S.A., 95:8892-8897.

91. Sobreira, C., M. Hirano, S. Shanske, R.K. Keller, R.G. Haller, E. Davidson, F.M. Santorelli, A.F. Miranda, E. Bonilla, D.S. Mojon, A.A. Barreira, M.P. King, S. DiMauro, 1997. Mitochondrial encephalomyopathy with coenzyme Q10 deficiency. Neurology, 48:1238-1243.

92. Ihara, Y., R. Namba, S. Kuroda, T. Sato, T. Shirabe, 1989. Mitochondrial encephalomyopathy (MELAS): pathological study and successful therapy with coenzyme Q10 and idebenone. J. Neurol. Sci., 90:263-271.

93. Chen, R.S., C.C. Huang, N.S. Chu, 1997. Coenzyme Q10 treatment in mitochondrial encephalomyopathies. Short-term double-blind, crossover study. Eur. Neurol., 37:212-218.

94. Sastre, J., A. Lloret, C. Borras, J. Pereda, D. Garcia-Sala, M.T. Droy-Lefaix, F.V. Pallardo, J. Vina, 2002. Ginkgo biloba extract EGb 761 protects against mitochondrial aging in the brain and in the liver. Cell Mol. Biol. (Noisy-le-grand), 48:685-692.

95. Perez-Pinzon, M.A., P.L. Mumford, M. Rosenthal, T.J. Sick, 1997. Antioxidants, mitochondrial hyperoxidation and electrical recovery after anoxia in hippocampal slices. Brain Res., 754:163-170.

96. Dolske, M.C., J. Spollen, S. McKay, E. Lancashire, L. Tolbert, 1993. A preliminary trial of ascorbic acid as supplemental therapy for 
autism. Prog. Neuropsychopharmacol. Biol. Psychiatry, 17:765-774.

97. Zilbovicius, M., N. Boddaert, P. Belin, J.B. Poline, P. Remy, J.F. Mangin, L. Thivard, C. Barthelemy, Y. Samson, 2000. Temporal lobe dysfunction in childhood autism: a PET study. Am. J. Psychiatry, 157:1988-1993.

98. Ohnishi, T., H. Matsuda, T. Hashimoto, T. Kunihiro, M. Nishikawa, T. Uema, M. Sasaki, 2000. Abnormal regional cerebral blood flow in childhood autism. Brain, 123:1838-1844.

99. Boddaert, N., M. Zilbovicius, 2002. Functional neuroimaging and childhood autism. Pediatr. Radiol., 32:1-7.

100. Rossignol, D.A, 2007. Hyperbaric oxygen therapy might improve certain pathophysiological findings in autism. Med. Hypotheses, 68:1208-1227.

101. Fatemi, S.H., A.R. Halt, 2001. Altered levels of $\mathrm{Bcl} 2$ and p53 proteins in parietal cortex reflect deranged apoptotic regulation in autism. Synapse, 42:281-284.

102. Araghi-Niknam, M., S.H Fatemi, 2003. Levels of Bcl-2 and P53 are altered in superior frontal and cerebellar cortices of autistic subjects. Cell Mol. Neurobiol., 23:945-952.

103. Graeber, T.G., J.F. Peterson, M. Tsai, K. Monica, A,J, Fornace Jr, A.J Giaccia, 1994. Hypoxia induces accumulation of p53 protein, but activation of a G1-phase checkpoint by lowoxygen conditions is independent of p53 status. Mol. Cell Biol., 14:6264-6277.

104. Shimizu, S., Y. Eguchi, W. Kamiike, Y. Itoh, J. Hasegawa, K. Yamabe, Y. Otsuki, H. Matsuda, Y. Tsujimoto, 1996. Induction of apoptosis as well as necrosis by hypoxia and predominant prevention of apoptosis by Bcl-2 and Bcl-XL. Cancer Res., 56:2161-2166.

105. Magalhães, J., A. Ascensão, J.M. Soares, R. Ferreira, M.J. Neuparth, F. Marques, J.A. Duarte, 2005. Acute and severe hypobaric hypoxia increases oxidative stress and impairs mitochondrial function in mouse skeletal muscle. J. Appl. Physiol., 99:1247-1253.

106. Calvert, J.W., J.H. Zhang, 2007. Oxygen treatment restores energy status following experimental neonatal hypoxia-ischemia. Pediatr. Crit. Care Med., 8:165-173.
107. Lane, N., 2002. Oxygen: the molecule that made the world. Oxford University Press, p. 166-167.

108. Dave, K.R., R. Prado, R. Busto, A.P. Raval, W.G. Bradley, D. Torbati, M.A. Perez-Pinzon, 2003. Hyperbaric oxygen therapy protects against mitochondrial dysfunction and delays onset of motor neuron disease in Wobbler mice. Neuroscience, 120:113-120.

109. Daugherty, W.P., J.E. Levasseur, D. Sun, G.L. Rockswold, M.R. Bullock, 2004. Effects of hyperbaric oxygen therapy on cerebral oxygenation and mitochondrial function following moderate lateral fluid-percussion injury in rats. $\mathbf{J}$. Neurosurg., 101:499-504.

110. Boveris, A., B. Chance, 1973. The mitochondrial generation of hydrogen peroxide: general properties and effect of hyperbaric oxygen. Biochem. J., 134:707-716.

111. Gosalvez, M., J. Castillo Olivares, E. De Miguel, M. Blanco, D. Figuera, 1973. Mitochondrial respiration and oxidative phosphorylation during hypothermic hyperbaric hepatic preservation. J. Surg. Res., 15:313-318.

112. Bar-Sagie, D., A. Mayevsky, B. Bartoov, 1981. Effects of hyperbaric oxygenation on spermatozoan motility driven by mitochondrial respiration. J. Appl. Physiol., 50:531-537.

113. Lou, M., Y. Chen, M. Ding, C.C. Eschenfelder, G. Deuschl, 2006. Involvement of the mitochondrial ATP-sensitive potassium channel in the neuroprotective effect of hyperbaric oxygenation after cerebral ischemia. Brain Res. Bull., 69:109116.

114. Gutsaeva, D.R., H.B. Suliman, M.S. Carraway, I.T. Demchenko, C.A. Piantadosi, 2006. Oxygen induced mitochondrial biogenesis in the rat hippocampus. Neuroscience, 137:493-504.

115. Rossignol, D.A., L.W. Rossignol, 2006. Hyperbaric oxygen therapy may improve symptoms in autistic children. Med. Hypotheses, 67:216-228. 